\title{
Combined Manual and Automatic Landmark Detection for Enhanced Surface Registration of Anatomical Structures: an Extensive Parameter Study for Femur and Clavicle
}

\author{
Sanne Vancleef ${ }^{\mathrm{a} *}$, Yannick Carette ${ }^{\mathrm{a}}$, Hans Vanhove ${ }^{\mathrm{a}}$, Joost R. Duflou ${ }^{\mathrm{a}}$, Ilse \\ Jonkers $^{\mathrm{b}}$, Jos Vander Sloten ${ }^{\mathrm{a}}$ \\ ${ }^{a}$ Department of Mechanical Engineering, KU Leuven, Leuven, Belgium, ${ }^{b}$ Department of \\ Movement Sciences, KU Leuven, Leuven, Belgium \\ * sanne.vancleef@kuleuven.be
}

Notes on contributors

Sanne Vancleef received master degrees in physiotherapy and rehabilitation sciences and biomedical engineering from KU Leuven in 2012 and 2015 respectively. Currently she is involved in a PhD programme in Biomedical engineering at KU Leuven. Her main research topic is the optimisation of thin plates for bone reconstruction and fracture fixation. To achieve this, she focuses on defining shape variations of human bones and the quantification of the muscle and joint reaction forces acting on these bones during activities of daily living.

Yannick Carette received a master degree in mechanical engineering in 2015. As $\mathrm{PhD}$ student, he is part of the sheet metal forming group at the department of mechanical engineering, led by professor Duflou. His main research focus is the improvement of the accuracy of the Single Point Incremental Forming (SPIF) process in the production of medical implants through the use of software based process planning. He works closely together with two researchers from the biomechanical and medical department on the BioMeTIOm project, which has as goal to identify and exploit the opportunities of the flexible SPIF forming process to be used in different medical applications.

Hans Vanhove holds a master degree in electro-mechanical engineering from KU Leuven since 2007. He is currently active as a research fellow and PhD candidate at the same university. His main topics are flexible CNC sheet metal processes, among which; incremental forming, laser cutting and bending. Focusing both on hardware and toolpath optimizations. In Incremental forming, as the centre of his research, he aims to improve accuracy and shape complexity for small batch sheet production. This research leads to new applications of metal sheet in patient tailored implants and custom design panels.

Joost Duflou holds master degrees in Architectural and Electro-mechanical Engineering and a $\mathrm{PhD}$ in Engineering Sciences from KU Leuven, Belgium. After a number of years of industrial experience in different international companies, he has been a faculty member at the Mechanical Engineering Department of KU Leuven since 1997. He became a tenured Full Professor in 2012. His principal research activities are situated in the field of design support methods and methodologies, with special attention for Ecodesign and Life Cycle Engineering, and Sustainable 
Manufacturing. As chairholder of the LVD Chair on Sheet Metal Processing, he also leads a research group focussing on sheet metal oriented manufacturing processes and systems and he supervises the KU Leuven FabLab. He is a CIRP Fellow and has published over 250 international publications. As chair and board member of several spin-off companies and professional associations he contributes to research valorisation and dissemination.

Ilse Jonkers received her $\mathrm{PhD}$ in 2000 from the University of Leuven (KU Leuven). After postdoctoral stays at the University of Stanford and KU Leuven, she began her career as an independent researcher in 2009 with an appointment as Assistant Professor with tenure in the Human Movement Biomechanics Research Group at KU Leuven. Currently, she is head of this research group. The majority of her research activities rely on the use of 3D motion analysis, personalized musculoskeletal modelling and multi-body simulation. Using these methodologies, she wants to further the understanding of the neuromuscular constraints of gross motor function and relate gross motor function, joint and tissue loading to musculoskeletal adaptation. Applications relate to the understanding of joint and implant loading during gait and the remediation of gait disorders in patients. With this research, she hopes to provide fundamental insights to optimize rehabilitation strategies and surgical interventions.

Jos Vander Sloten obtained his MSc and $\mathrm{PhD}$ in mechanical engineering from KU Leuven in 1985 and 1990 respectively. Currently he is full professor within the Division of Biomechanics at KU Leuven. He founded the Leuven Medical Technology Centre (L-MTC) in 2008 and served as the first chairman for two terms until 2016. His teaching assignments are engineering mechanics, problem solving and engineering design, computer integrated surgery systems. His research interests are computer applications in musculoskeletal biomechanics and computer integrated surgery, on which he authored more than 160 journal papers. He is member of the council of the Belgian Society for Medical and Biological Engineering and Computing, and a former council member of the European Society of Biomechanics. In the European Alliance for Medical and Biological Engineering and Science (EAMBES) he served as secretary-general (2003-2004), president-elect (2005) and president (2006). He was elected Founding Fellow of EAMBES and more recently also as a Fellow of the International Academy for Medical and Biological Engineering. Since 2014 he represents the Faculty of Engineering Science of KU Leuven within the Board of Directors of CESAER, the Conference of European Schools of Advanced Engineering Education and Research. Since 2016 he is the Vice Dean for International Affairs of the Faculty of Engineering Science, KU Leuven. 


\title{
Combined Manual and Automatic Landmark Detection for Enhanced Surface Registration of Anatomical Structures: an Extensive Parameter Study for Femur and Clavicle
}

\author{
Surface registration, in which a mapping between surfaces is calculated, is a powerful tool \\ to create patient-specific musculoskeletal and statistical shape models. However, to create \\ these models, surface registration must assure a one-to-one correspondence between both \\ surfaces. To enhance this process, a surface registration framework that uses a combination \\ of manual and automatic landmark detection is presented in this study. In addition, an \\ extensive parameter study of the framework for a femur and a clavicle is conducted. The \\ average correspondence quality of nine femur landmarks decreased from $15.6 \mathrm{~mm}$ using \\ automatic point detection to $6.1 \mathrm{~mm}$ by combining manually and automatically indicated \\ landmarks. For the clavicle, the average distance of five landmarks decreased from 2.8 to \\ $0.9 \mathrm{~mm}$. Combining manual and automatic landmark detection clearly improved \\ correspondence quality. Results confirmed the applicability of the proposed registration \\ framework for femur and clavicle, although with different parameter settings.
}

Keywords: Surface registration, non-rigid registration, clavicle, femur

\section{Introduction}

Complex biomechanical problems are commonly addressed by generic models representing the anatomy of an average adult. Although such generic models are frequently used to analyse the functional outcome after surgery (Reinbolt et al. 2009), and the effect of disorders such as cerebral palsy (Arnold et al. 2001) and osteoarthritis (Meireles et al. 2016) on the musculoskeletal system, patient-specific musculoskeletal models are expected to be more suitable to study these problems (Lenaerts et al. 2008). One way to create these patient-specific models is to register a generic bone surface mesh with known muscle attachment sites to the patient-specific bone mesh using surface registration. 
Furthermore, surface registration is also the first step in the creation of statistical shape models. These models are a powerful tool for describing bone shape variation (Lu \& Untaroiu 2013),(Zhang et al. 2014), 3D-data reconstruction from 2D images (Tsai et al. 2015), facilitation of segmentation (Heimann \& Meinzer 2009) and implant design (Kozic et al. 2010). The latter is particularly relevant for clavicle fracture plates, since current plates have a poor geometric fit due to the complex S-shape of the clavicle (Huang et al. 2007),(Malhas et al. 2016). The use of a clavicle statistical shape model could have an added value in the design of better fitting plates.

During surface registration, a mapping which transforms one surface mesh to another is calculated. In these algorithms, each point of the source mesh is registered to its corresponding point in the target mesh. Corresponding points are found by geometric feature matching (Wang et al. 2002) or closest points (Amberg et al. 2007). Amberg et al proposed the non-rigid optimal step iterative closest point (N-ICP-T) algorithm. This N-ICP-T algorithm is an elasticity modulated registration in which each vertex is translated separately, but with constraints. This method achieves a good geometric fit, but often the final correspondences are poor (Danckaers et al. 2014). However, the creation of both patient-specific musculoskeletal models and statistical shape models requires surface registration that assures one-to-one correspondence. Therefore, Danckaers et al. (Danckaers et al. 2014) proposed the RN-ICP-T algorithm, which combines a rigid registration with the N-ICP-T algorithm. Their proposed algorithm is an iterative algorithm in which the global or rigid registration is most important in the first iteration and becomes less important in later iterations. During these later iterations, local deformations using the N-ICP-T algorithm gain importance.

However, this approach still suffers from two drawbacks. First, corresponding points are identified automatically based on the normal directions of source and target, which can lead to 
false positives. Second, the registration framework relies on setting multiple parameters, which all influence registration quality.

The goal of this study was to provide a registration framework that combines and extends different algorithms in order to further improve mesh correspondences. An extensive parameter study is performed for its use in registering a human femur and clavicle. Both cases have a completely different geometry -the femur containing different distinct tubercles, while the clavicle exhibits large variations in its s-shape. The high number of anatomical landmarks on the femur make this bone an ideal test case, whereas the limited number of anatomical landmarks on the clavicle allows to assess the algorithm's robustness. Combined, these bones thus provide suitable datasets for the validation of the generic nature and applicability of the proposed registration framework.

\section{Materials and Methods}

\subsection{Data}

One source and two target bones were selected for femur and clavicle. One of the targets was used to perform the parameter study the other target was used as a case study to test the identified optimal parameter set for each bone. The femur source geometry was obtained from the geometry of OpenSim (Delp et al. 2007), since muscle attachments are known and can be used in the future to create patient-specific musculoskeletal models through surface registration. The target geometries were taken from two patient-specific musculoskeletal CT models (Wesseling et al. 2016). Out of a database of 49 clavicle CT scans, a source and two targets were defined by random selection and segmented using Mimics (Materialise, Belgium). Both source meshes were remeshed using 3-matic (Materialise, Belgium) such that a uniform mesh was obtained (femur edge length: $2 \mathrm{~mm}$, clavicle edge length: $1 \mathrm{~mm}$ ). The femur mesh consisted of 
14626 vertices and the clavicle mesh of 10541 vertices. The longitudinal length of the source femur was $452.3 \mathrm{~mm}$ and the target femur used for the parameter study had a length of $492.4 \mathrm{~mm}$. The length was measured by first calculating the inertia axis of each bone (Small Satellites 2012). Then, the bone and its inertia axes were aligned with the world coordinate system and the bone length was calculated as the distance between the maximal and minimal value along the longitudinal axis using Matlab (Mathworks, USA). The lesser trochanter centre was located at $17.5 \%$ and $20.1 \%$ of the femurs' length respectively. The source clavicle length was $152.8 \mathrm{~mm}$ and the target clavicle used for the parameter study length was $137.3 \mathrm{~mm}$. The conoid tubercle was located at $20.2 \%$ and $19.8 \%$ of the clavicles' length respectively.

To evaluate the influence of manually identified landmarks on the registration, nine landmarks were manually identified on the source and target femurs: medial epicondyle (MEP), lateral epicondyle (LEP), most posterior point of medial and lateral condyle (MCP and LCP) (Victor et al. 2009), the adductor tubercle, the lesser trochanter, the lesser trochanter upper and lower transition and the upper side of the greater trochanter (Figure 1A, B and C) (Valente et al. 2014)(Zhang et al. 2014). For the clavicle, five landmarks were manually identified on the source and target clavicle (Figure 1D), the conoid tubercle, the most ventral (Wu et al. 2005) and inferior point of the sternoclavicular joint (SCJ) and the most ventral and dorsal (Wu et al. 2005) point on the acromioclavicular joint (ACJ).

\subsection{Algorithm}

Figure 2 provides an overview of the surface registration framework. To build this framework, existing algorithms are used for the rigid registration (Besl \& McKay 1992) and the N-ICP-T algorithm (Amberg et al. 2007). Rigid registration starts with the ICP algorithm (Besl \& McKay 1992) which aligns the source and target mesh by iteratively minimising the distance between 
two surfaces. During each iteration, closest points from one mesh to the other are identified and the distance between these points is minimised by calculating and applying a translation and rotation matrix. To further decrease the distance from source to target mesh and facilitate corresponding point search in subsequent steps, similar length of source and target mesh is preferable. Therefore, uniform mesh scaling is implemented as additional part of the rigid registration. A scale factor is determined by dividing the longitudinal target length by the longitudinal source length.

Second, elasticity modulated registration is applied, consisting of a two-step iterative process. (1) In the first step, corresponding points are identified either by manually entering the vertex indices of corresponding points of source and target mesh or by automatically identifying the corresponding points. Apart from the first iteration, this automatic corresponding point search can be skipped during following iterations. In this case, corresponding points from the previous iteration will be used. Automatic corresponding point detection is based on ray tracing, in which a line along each source's vertex normal is drawn. The vertex normal is computed using the normal of neighbouring faces (Max 1999). A possible corresponding point is found at the intersection of this line with the target mesh. Next, the dot product of the normalised vertex normal and the normal of the face containing the intersection point is calculated. If the dot product exceeds a predefined threshold, for the dot product being between 0 and 1 , these points are considered to be valid corresponding points. In case of multiple valid correspondences with the target, the intersection point with the smallest Euclidean distance is considered. In case of manually identifying corresponding points, an automatic corresponding point search is still conducted to achieve global mesh registration for areas without nearby landmarks. However, the automatically identified corresponding points for the manual landmarks are replaced by the 
manually entered vertex indices, and the corresponding points for the vertices surrounding the manually indicated landmarks are cleared. These corresponding points then become input for the next step in the registration framework. (2) In the second step, the iterative N-ICP-T algorithm, described by Amberg et al. (Amberg et al. 2007), is used to control the mapping from source to target. The N-ICP-T algorithm consists of two loops: the inner and outer loop. In the outer loop, the stiffness value decreases. This decrease is defined by formula (1) and is dependent on the number of iterations of the outer loop and the start and end stiffness:

$$
\text { stiffness }^{n}=\text { stiffness }^{n-1}-\frac{\text { stiffness }^{\text {start }}-\text { stiffness }^{\text {end }}}{\text { number of outer iterations-1 }}
$$

The stiffness term is a regulariser which minimises the difference between translations of neighbouring vertices. This stiffness value decrease results in a global scaling behaviour in the first iterations, followed by more local deformations in later iterations. During the inner loop, the source is deformed by translating corresponding point towards each other, while relative motion of neighbouring vertices is restricted by the stiffness value (Amberg et al. 2007). After registration, the error of the corresponding points from target to deformed source is calculated by the following formula:

$$
\text { Error }=\frac{\sum_{i=1}^{n} \mid \text { corresponding point target }{ }^{i} \text {-corresponding point source }{ }^{i} \mid}{n}
$$

This error is then compared to the error from the previous loop. In case the difference between both is smaller than $\mathcal{E}(0.1 \mu \mathrm{m}$, which is a compromise between accuracy and calculation time $)$, the deformation of the source mesh is considered to be converged and a new iteration in the outer loop can start. 
The registration framework thus depends on a number of specific parameters: the dot product threshold, the procedure for corresponding points detection, whether or not corresponding point search is conducted during each iteration, start and end stiffness of the N-ICP-T algorithm, as well as the number of outer iterations. By default, the end stiffness is set to 2 , the dot product to 0.5 , the number of outer iterations for femur to 40 and for the clavicle to 10 , and corresponding point search is performed each outer iteration. During the tests, one parameter was varied at a time while maintaining the other parameters at the default settings. End stiffness varied from 0 to 100 , the dot product threshold from 0 to 0.9 , the number of outer iterations from 5 to 100 , and execution of corresponding point search varied from each to every $2,3,4,5,10$ and 20 outer iterations for the femur and from each to every 2, 3 and 5 outer iterations for the clavicle.

\subsection{Evaluation of algorithm}

The effect of these parameter settings on computational time, the geometric error, correspondence and mesh quality of the registered mesh was investigated. Computational time was measured using the tic toc function in Matlab and expressed in minutes. Geometric error was defined as the average distance of all target vertices to their closest source point. Correspondence quality was evaluated using the absolute distances between the original landmark vertices of the target and the landmark vertices of the deformed source. The average and individual behaviour of the landmarks were examined. Furthermore, correspondence quality between manual and automatic landmark detection was compared. Mesh quality was evaluated as the percentage of the number of overlapping and intersecting triangles in the deformed source as assessed using 3-matic (Materialise, Belgium), which provides a measure for mesh continuity.

Registration results in this study were obtained with Matlab on a PC with a quad core second generation Intel core i5 processor and 8GB of RAM. 


\section{Results}

\subsection{Effect of parameters}

\subsubsection{Time}

As shown in Figure 3A, computational time increased linearly with the number of outer iterations. End stiffness and the threshold for corresponding points have limited influence on the time (Figure 3B, C). Figure 3D shows that computation time can drastically be decreased by omitting the corresponding point detection in multiple iterations.

\subsubsection{Automatic corresponding point detection}

The geometric error of the femur converged after 10 outer iterations (Figure 4A). However, the average distance between landmarks decreased with subsequent iterations (Figure 4B). The latter also holds for the clavicle, but the geometric error remained constant regardless of the number of outer iterations (Figure 4A). Mesh quality is influenced by the number of outer iterations as can be seen in Figure 4C. Based on these observations, 40 iterations for the femur and 10 iterations for the clavicle were used for further analysis. Figure 5 shows the evolution of the geometric error through the registration process of the femur with 40 outer iterations. After 10 outer iterations, the geometric error was $0.3330 \mathrm{~mm}$, decreasing to $0.0030 \mathrm{~mm}$ after 40 iterations. [Figure 5 near here]

A lower end stiffness resulted in a smaller geometric error in both femur and clavicle (Figure 4D). Corresponding point quality improved with higher end stiffness in femur and clavicle, however the improvement in the clavicle is only $0.1 \mathrm{~mm}$ (Figure 4E). As can be seen in Figure 4F, end stiffness did not affect the mesh quality. 
The dot product threshold had limited influence on the geometric error. Only for a threshold of 0.9 the geometric error of the femur increased (Figure 4G). The clavicle geometric error ranged from 2.6e-3 to 2.7e-3mm. However, a larger threshold resulted in a better correspondence quality for the femur as well as for the clavicle (Figure 4H). Again, no influence on the mesh quality was observed (Figure 4I).

As can be seen in Figure 4J and 4K, increasing the number of corresponding point searches resulted in a lower geometric error and higher correspondence quality. A lower number of executions resulted in poor mesh quality (Figure 4L).

The behaviour of the individual landmarks can be found in the supplementary material.

\subsubsection{Combined automatic and manual corresponding point detection}

Figure 6 shows the number of iterations for which manual landmarks of the femur were used as corresponding points. For the remaining iterations, automatic corresponding point searches were conducted. The use of manually identified landmarks for all outer iterations resulted in a geometric error of $0.08 \mathrm{~mm}$ and a perfect correspondence quality (Figure 6A, B). Mesh quality is inferior when using the manual landmarks for more than 20 iterations (Figure 6C). However, the average correspondence quality improved from $15.6 \mathrm{~mm}$ in case 40 iterations of automatic corresponding point detection were used (Fig 3B), to $7.4 \mathrm{~mm}$ in case 5 iterations of manual corresponding points were used, followed by 35 iterations of automatic corresponding point detection (Fig 6B). The improvement in correspondence quality for the femur landmarks using the combination of manually and automatically indicated corresponding points compared to using only automatic corresponding point detection can be seen in table 1.

A similar analysis is made for the clavicle. Figure 7 shows the number of outer iterations in which manual landmarks are used. For the remaining outer iterations, automatic corresponding 
point detection was used. Using manual correspondences in all iterations led to a geometric error of $0.0030 \mathrm{~mm}$ and an inferior mesh quality (Figure 7A, C). However, an excellent correspondence quality was achieved (Figure 7B). Using the combination of automatically and manually identified corresponding points improved correspondence quality for ACJ dorsal, SCJ ventral, conoid tubercle, ACJ ventral and SCJ inferior, from 3.7, 2.9, 1.5,4.1 and $2.1 \mathrm{~mm}$, to 1, $1.4,0.1,0.9$ and $1.2 \mathrm{~mm}$ respectively using nine outer iterations with manually identified landmarks, which is the maximum resulting in a mesh without intersecting and overlapping triangles.

\subsection{Case study with optimal parameter settings}

Registration of a second target femur mesh with the optimal parameters from the parameter study ( 40 iterations, end stiffness $=2$, and threshold of 0.7 ) yielded a geometric error of $0.0030 \mathrm{~mm}$ and an average correspondence quality of $5.2 \mathrm{~mm}$. Using manual landmarks for 20 out of 40 iterations, resulted in a geometric error of $0.0030 \mathrm{~mm}$ and an average correspondence quality of $3.6 \mathrm{~mm}$. For the clavicle, using the optimal parameters for another clavicle study (10 iterations, end stiffness $=2$, and threshold of 0.8 ) resulted in a geometric error of $0.0026 \mathrm{~mm}$ and an average correspondence quality of $3.2 \mathrm{~mm}$. Using manual landmarks for 8 out of 10 iterations resulted in a geometric error of $0.0028 \mathrm{~mm}$ and an average correspondence quality of $1.8 \mathrm{~mm}$.

\section{Discussion}

In this study, an enhanced surface registration framework was developed and an extensive parameter study was performed. The input of manually indicated landmarks clearly improved correspondence quality, which is an important criterion to develop patient-specific musculoskeletal and statistical shape models. Furthermore, the registration framework reduced 
the average geometric error compared to Danckaers (Danckaers et al. 2014) in case of the clavicle. The geometric error in Danckaers was $0.065 \mathrm{~mm}( \pm 0.007)$, while in this study, the geometric error was $0.0026 \mathrm{~mm}$ for 10 outer iterations. In case of optimal parameter settings, this registration framework is thus generic and applicable to both femur and clavicle. The parameter study revealed parameter dependency on the shape of the bone. Indeed, clavicle and femur are geometrically two very different bones; the clavicle has more variations in the s-shape, while the femur has more tubercles. Despite this, our analysis indicates the general applicability of the registration method to bones of different shapes.

Our results show a clear trade-off between computational time and registration accuracy. Overall, computational time in this study was in the same order of magnitude as in Danckaers et al. However, absolute time comparison is difficult since the computer specifications were not mentioned in Danckaers et al. The most time consuming step of the registration framework is the corresponding point search. Hence, reducing the execution of the correspondence detection algorithm drastically influences computational time. However, conducting the correspondence detection algorithm in each iteration improved correspondence quality and decreased geometric error. In case points are forced to migrate to a specific point during several iterations, the mesh gets less deformation freedom, thereby complicating corrections of an erroneously migrated point. In addition, mesh quality is inferior. Therefore, corresponding point search is recommended in each outer iteration (Figure 4J,K,L). To select the suitable values for the other parameters, geometric error, correspondence quality and mesh quality should be taken into account.

Using manually identified corresponding points in the registration framework drastically improved correspondence quality, as it prevents erroneous initial correspondences. The average 
distance from the nine femur landmarks decreased from $15.6 \mathrm{~mm}$ using automatic corresponding point detection to $6.1 \mathrm{~mm}$ using manual landmarks in 20 of the 40 outer iterations. If the manual identified landmarks are used during too many outer iterations, mesh quality is inferior because those points are forced to migrate to a certain point thereby losing mesh continuity. Therefore, once the landmarks are migrated in the right direction, automatic corresponding point search should be conducted.

Dai et al. reported an average intra user variability of $2.9 \mathrm{~mm}$ and a maximum deviation of up to $6.3 \mathrm{~mm}$ (Dai et al. 2014). In case of the clavicle, the correspondence quality is already within intra-user variability, therefore the manual identification of landmarks in the clavicle could be omitted. Looking at the correspondence quality of both target femurs in case manual landmarks are used, the correspondence quality is in the same order of magnitude. Although manually identifying these corresponding points is more time consuming than automatically detecting them, the improvement in correspondence quality is important to create patient-specific musculoskeletal models. The major and minor trochanter and adductor tubercle are important sites for muscle attachments. Outcome of the musculoskeletal model depends on these muscle attachment sites (Carbone et al. 2012), muscle direction and moment arm (Lenaerts et al. 2008). Therefore, correct prediction of muscle attachment sites through registration is important and time to manually indicate landmarks can be justified.

A better initial match between source and target leads to better correspondence quality, as is shown by the better correspondence quality using only automatic point detection in the second target femur. In the target femur used for the parameter study, the adductor tubercle and MCP showed good agreement with automatic landmark detection. Correspondence quality of the other landmarks improved by adding manually identified corresponding points. This can be due to the 
position of the lesser trochanter in source and target mesh. As described earlier, the centre of the lesser trochanter is located at $17.5 \%$ of the length in the source and at $20.1 \%$ in the target. This makes the identification of true correspondences harder. In the clavicle, the location of the conoid tubercle in the source and target mesh is similar and therefore the correspondence quality is better.

Several limitations of this study should be kept in mind when interpreting results. First, for both applications, the behaviour for only one target was examined. Additional targets may show different geometric error and correspondence quality trends, since an increased geometric similarity between source and target results in better registration quality. However, conducting an extensive parameter study for one case provides a good initial estimate of registration parameters as shown by the case study. Parameters could then be altered for the inferiorly registered targets. Second, relating differences in accuracy between femur and clavicle to the subject's anthropometrics was impossible, since sex of the source femur and age, body weight and height of the target meshes were unknown. Third, the number of vertices of both sources is different, making comparison between both cases difficult, as the stiffness effect is dependent on the number of vertices (Amberg et al. 2007). The average length of the male right clavicle is 151mm (Parsons FG. 1916) while the femur is 443.6mm (Rubin et al. 1992). Therefore, down sampling both sources to a similar number of vertices would not make sense either.

Future research will therefore focus on creating a mesh independent registration framework. In addition, differences between the registered OpenSim muscle attachment sites and the patient-specific muscle attachment sites from MRI will be investigated.

In conclusion, this paper proposes an enhanced surface registration framework and conducts an extensive parameter study. Results show a superior correspondence when using 
manual landmarks and confirm the applicability of the proposed registration framework for meshes with different geometrical properties, although with different parameters. Therefore, it is recommended to conduct an extensive parameter study for one target, which can then be used for all other targets. 
Acknowledgements. The authors would like to thank the Materialise Chair for access to Mimics and 3matic, Walter Coudyzer of the radiology department of the University Hospital Leuven to provide us the CT scans from which the clavicles were segmented, and Mariska Wesseling for providing us the femur STL files.

Disclosure statement. No potential conflict of interest was reported by the authors.

Funding details. Funding was provided through internal funding of KU Leuven (BioMeTIOm IDO project).

\section{References}

Amberg B, Romdhani S, Vetter T. 2007. Optimal step nonrigid ICP algorithms for surface registration. Proc IEEE Comput Soc Conf Comput Vis Pattern Recognit.:0-7.

Arnold AS, Blemker SS, Delp SL. 2001. Evaluation of a deformable musculoskeletal model for estimating muscle-tendon lengths during crouch gait. Ann Biomed Eng. 29:263-274.

Besl P, McKay N. 1992. A Method for Registration of 3-D Shapes. IEEE Trans Pattern Anal Mach Intell. 14:239-256.

Carbone V, van der Krogt MM, Koopman HFJM, Verdonschot N. 2012. Sensitivity of subjectspecific models to errors in musculo-skeletal geometry. J Biomech. 45:2476-2480.

Dai Y, Seebeck J, Henderson AD, Bischoff JE. 2014. Influence of landmark and surgical variability on virtual assessment of total knee arthroplasty. Comput Methods Biomech Biomed Engin. 17:1157-1164.

Danckaers F, Huysmans T, Lacko D, Ledda A, Verwulgen S, Van Dongen S, Sijbers J. 2014. Correspondence Preserving Elastic Surface Registration with Shape Model Prior. Pattern Recognit (ICPR), 22nd Int Conf.:2143-2148.

Delp SL, Anderson FC, Arnold AS, Loan P, Habib A, John CT, Guendelman E, Thelen DG. 2007. OpenSim: Open-source software to create and analyze dynamic simulations of movement. IEEE Trans Biomed Eng. 54:1940-1950.

Heimann T, Meinzer HP. 2009. Statistical shape models for 3D medical image segmentation: A review. Med Image Anal. 13:543-563.

Huang JI, Toogood P, Chen MR, Wilber JH, Cooperman DR. 2007. Clavicular anatomy and the applicability of precontoured plates. J Bone Joint Surg Am. 89:2260-2265.

Kozic N, Weber S, Büchler P, Lutz C, Reimers N, González Ballester MÁ, Reyes M. 2010. Optimisation of orthopaedic implant design using statistical shape space analysis based on level sets. Med Image Anal. 14:265-275.

Lenaerts G, De Groote F, Demeulenaere B, Mulier M, Van der Perre G, Spaepen A, Jonkers I. 2008. Subject-specific hip geometry affects predicted hip joint contact forces during gait. J Biomech. 41:1243-1252.

Lu Y-C, Untaroiu CD. 2013. Statistical shape analysis of clavicular cortical bone with 
applications to the development of mean and boundary shape models. Comput Methods Programs Biomed. 111:613-628.

Malhas AM, Skarparis YG, Sripada S, Soames RW, Jariwala AC. 2016. How well do contoured superior midshaft clavicle plates fit the clavicle? A cadaveric study. J Shoulder Elb Surg. 25:954-959.

Max N. 1999. Weights for Computing Vertex Normals from Facet Normals. J Graph Tools. 4:16.

Meireles S, De Groote F, Reeves ND, Verschueren S, Maganaris C, Luyten F, Jonkers I. 2016. Knee contact forces are not altered in early knee osteoarthritis. Gait Posture. 45:115-120.

Parsons FG. 1916. On the Proportions and Characteristics of the Modern English Clavicle. J Anat. 51(Pt1):71-93.

Reinbolt JA, Fox MD, Schwartz MH, Delp SL. 2009. Predicting outcomes of rectus femoris transfer surgery. Gait posture. 30:100-105.

Rubin P, Leyvraz P, Aubaniac J, Argenson J, Esteve P, de Roguin B. 1992. The morphology of the proximal femur. A three-dimensional radiographic analysis. J Bone Jt Surg Br. 74:28-32.

Small Satellites. 2012. Moments of inertia, center of mass [Internet]. [cited 2018 Mar 19]. Available from: https://nl.mathworks.com/matlabcentral/fileexchange/39371-moments-ofinertia-center-of-mass?focused $=3776583 \&$ tab $=$ function

Tsai T-Y, Li J-S, Wang S, Li P, Kwon Y-M, Li G. 2015. Principal component analysis in construction of 3D human knee joint models using a statistical shape model method. Comput Methods Biomech Biomed Engin. 18:721-729.

Valente G, Pitto L, Testi D, Seth A, Delp SL, Stagni R, Viceconti M, Taddei F. 2014. Are subject-specific musculoskeletal models robust to the uncertainties in parameter identification? PLoS One. 9(11):e112625.

Victor J, Van Doninck D, Labey L, Innocenti B, Parizel PM, Bellemans J. 2009. How precise can bony landmarks be determined on a CT scan of the knee? The Knee. 16:358-365.

Wang YJ, Chua CS, Ho YK. 2002. Facial feature detection and face recognition from 2D and 3D images. Pattern Recognit Lett. 23:1191-1202.

Wesseling M, De Groote F, Bosmans L, Bartels W, Meyer C, Desloovere K, Jonkers I. 2016. Subject-specific geometrical detail rather than cost function formulation affects hip loading calculation. Comput Methods Biomech Biomed Engin. 19:1475-1488.

Wu G, Van Der Helm FCT, Veeger HEJ, Makhsous M, Van Roy P, Anglin C, Nagels J, Karduna AR, McQuade K, Wang X, et al. 2005. ISB recommendation on definitions of joint coordinate systems of various joints for the reporting of human joint motion - Part II: Shoulder, elbow, wrist and hand. J Biomech. 38:981-992.

Zhang J, Malcolm D, Hislop-Jambrich J, Thomas CDL, Nielsen PMF. 2014. An anatomical region-based statistical shape model of the human femur. Comput Methods Biomech Biomed Eng Imaging Vis. 2:176-185. 
A.

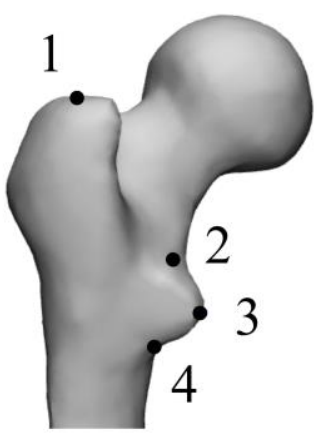

B.

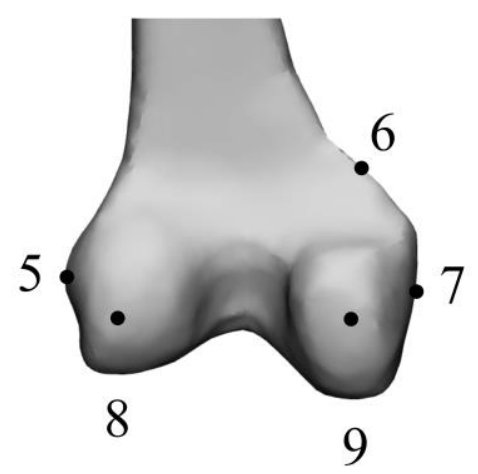

C.

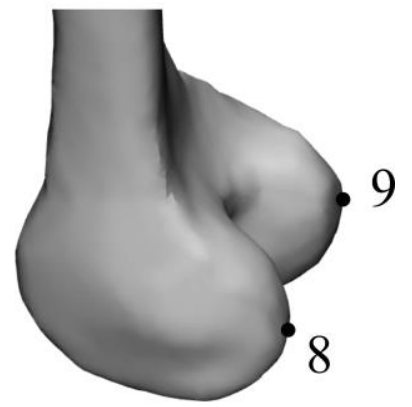

D.

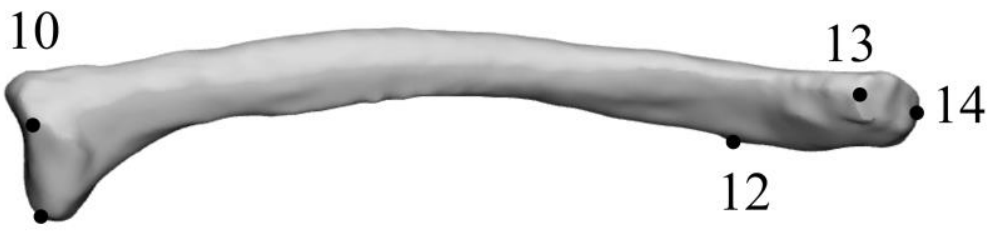

11

Figure 1. Landmarks of femur (A, B,C) and clavicle (D).1. Upper side of the greater trochanter 2. Lesser trochanter upper transition 3. Lesser trochanter 4. Lesser trochanter lower transition 5. Lateral epicondyle (LEC) 6. Adductor tubercle 7. Medial epicondyle (MEC) 8. Lateral condyle posterior (LCP) 9. Medial condyle posterior (MCP) 10. SCJ ventral 11. SCJ inferior 12. Conoid tubercle 13. ACJ ventral 14. ACJ dorsal 


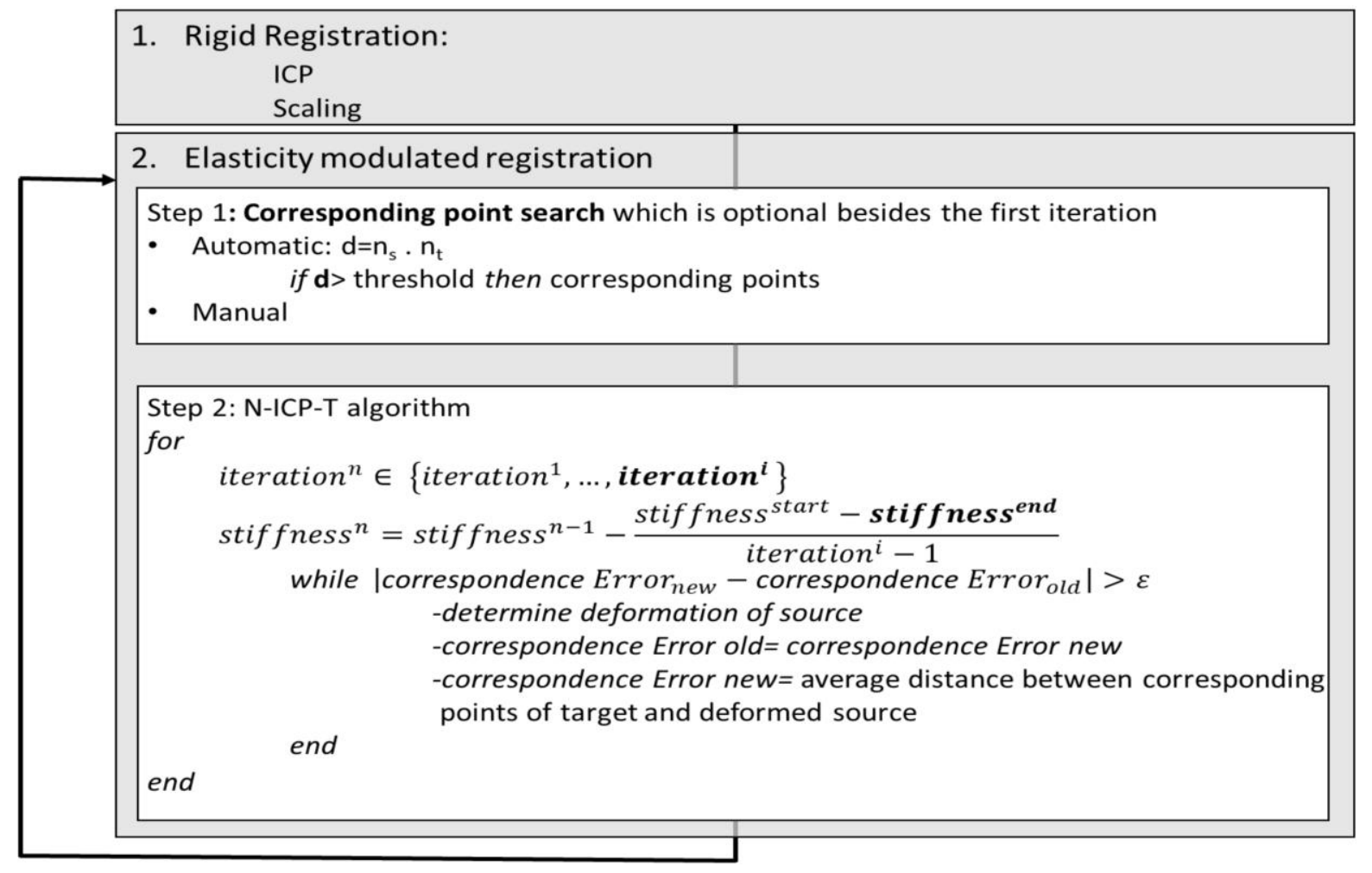

Figure 2. Surface registration framework in which the investigated parameters are indicated in bold. $\mathrm{D}$ is the dot product of the source vertex normal and the normal of the intersecting target face. The outer loop of the N-ICP-T algorithm corresponds to the for loop, the inner loop consists of the while loop. 

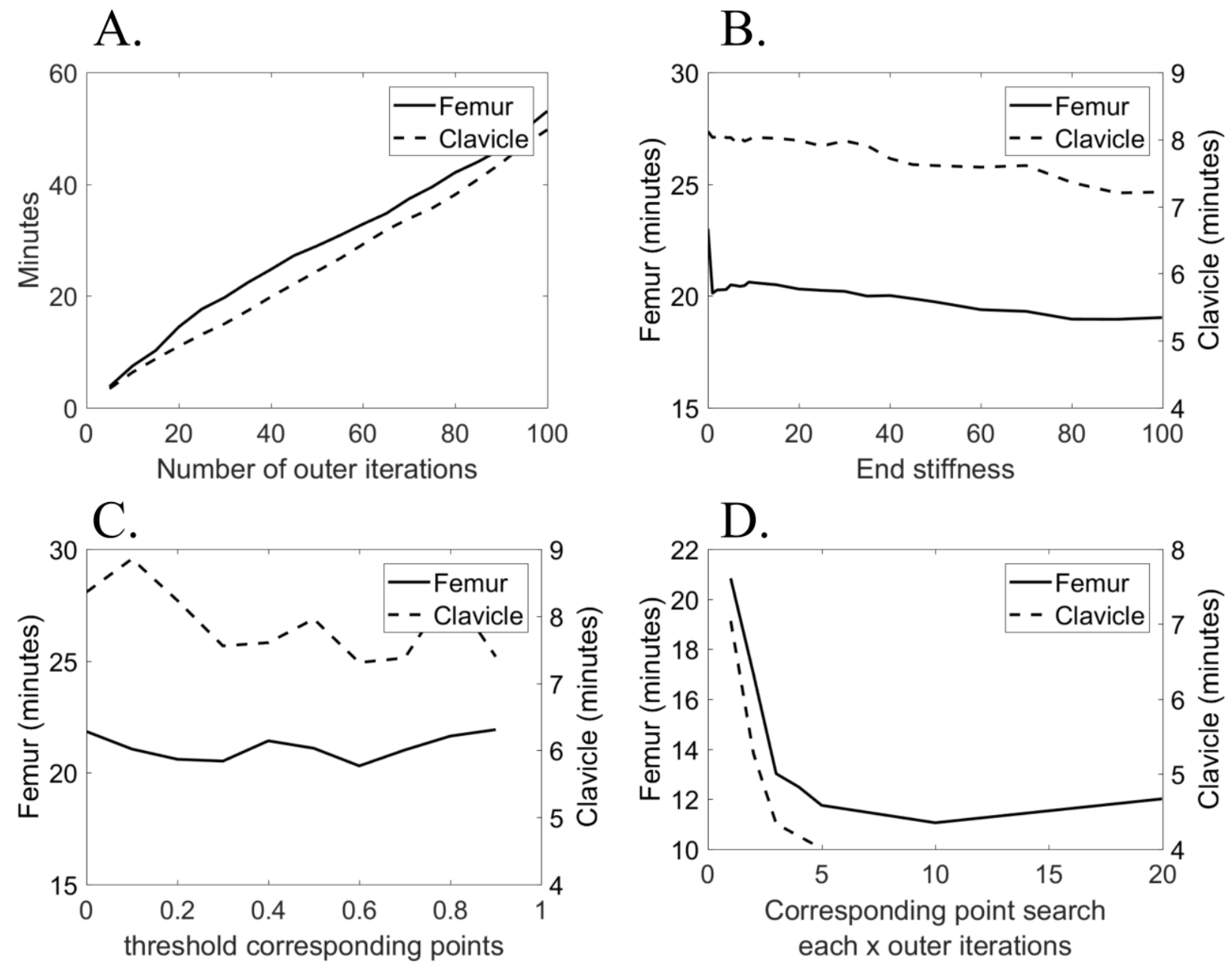

Figure 3. Influence of each parameter on computational time (minutes). Computational time in Figures 3B,C and D was evaluated for 40 outer iterations in case of the femur and 10 outer iterations in case of the clavicle. 


\section{Geometric error}

A.

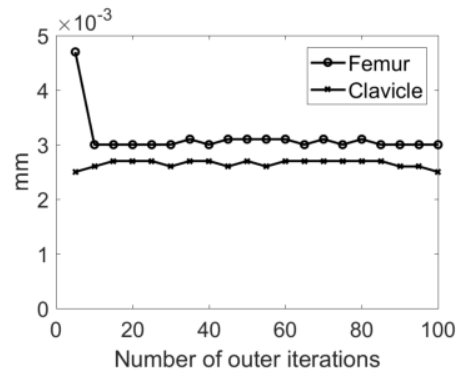

D.

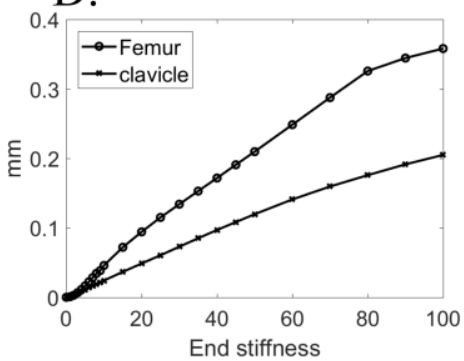

G.
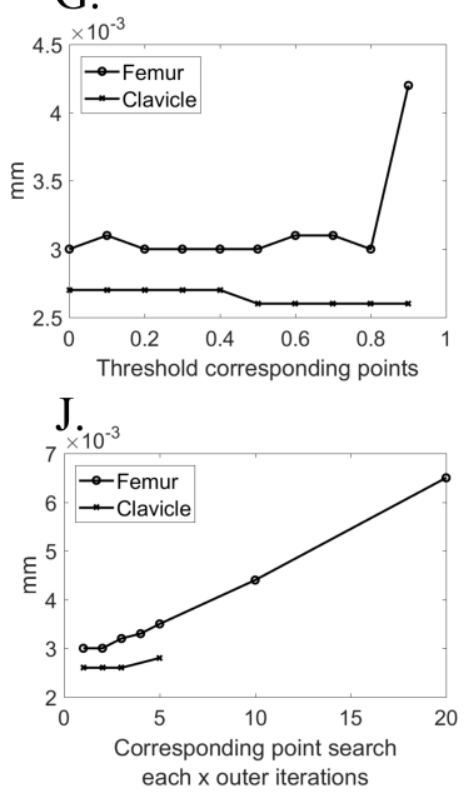

Correspondence quality

B.

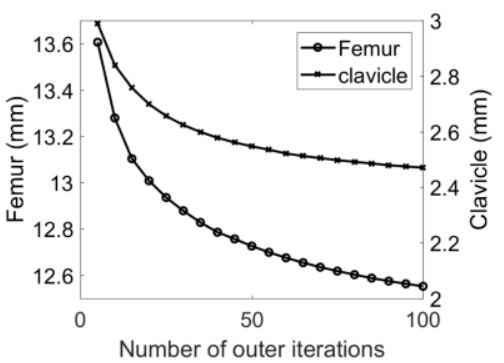

E.

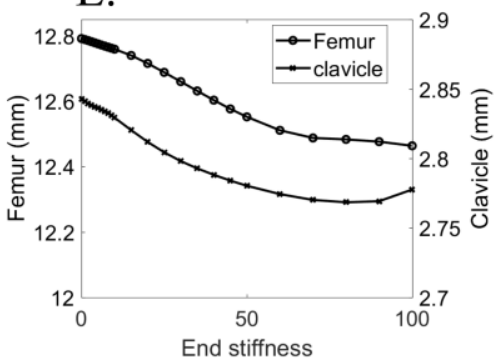

$\mathrm{H}$.

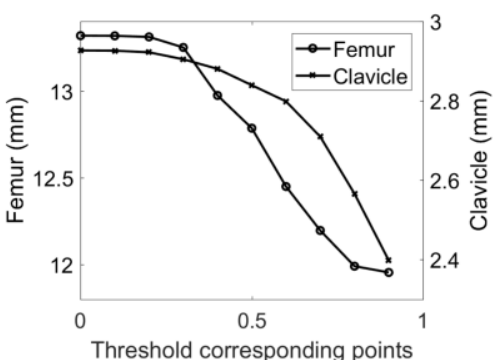

K.

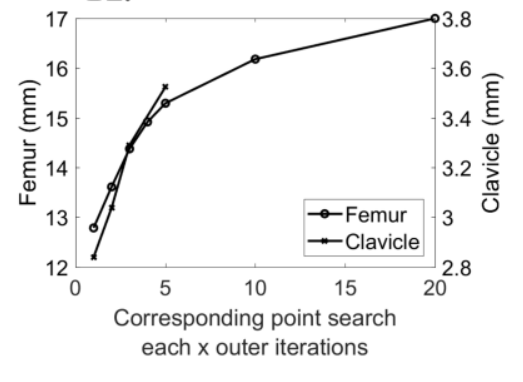

Mesh quality

C.

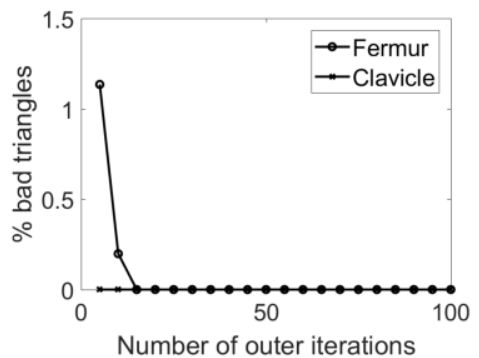

F.

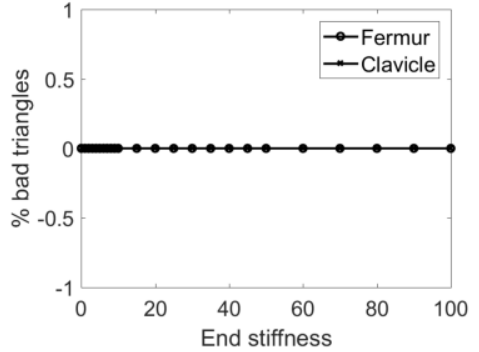

I.

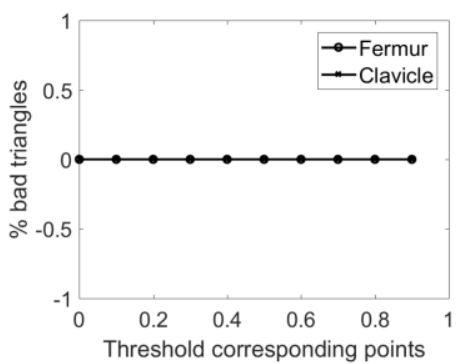

L.

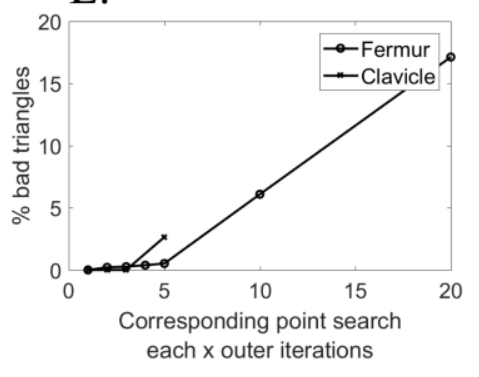

Figure 4. Results of different parameters on the geometric error, correspondence quality and mesh quality. 


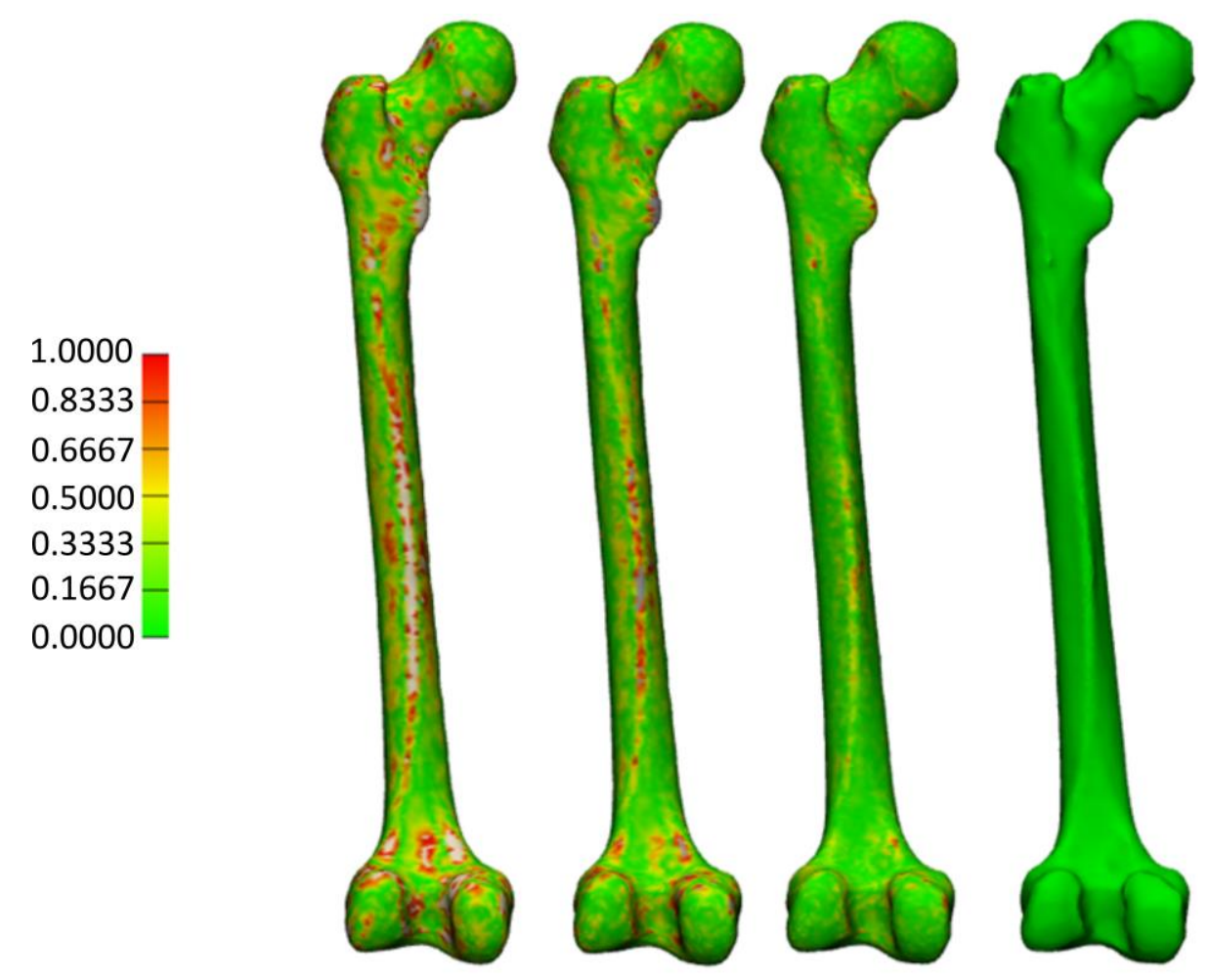

Figure 5. From left to right: evolution of geometric error (mm) after 10, 20, 30 and 40 out of 40 outer iterations using automatic corresponding point detection. 
A.

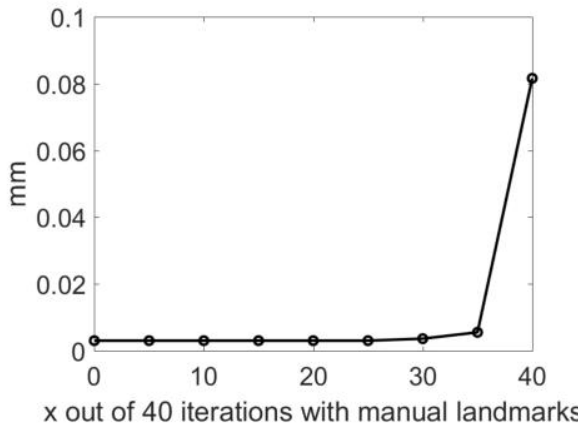

C.

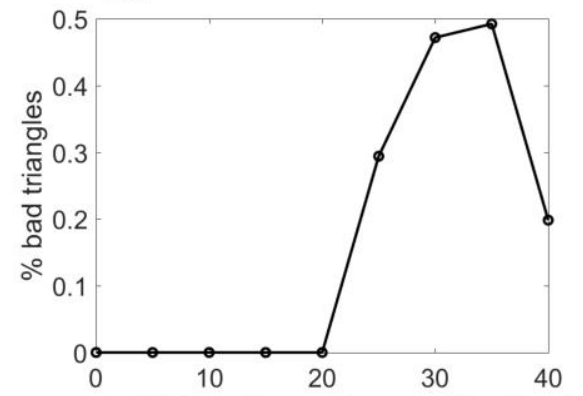

$\mathrm{x}$ out of 40 iterations with manual landmarks
B.

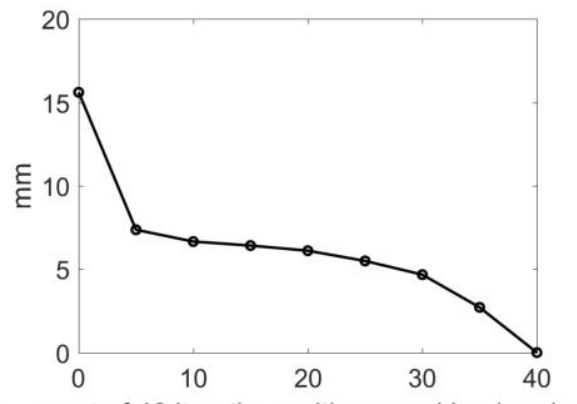

$x$ out of 40 iterations with manual landmarks

D.

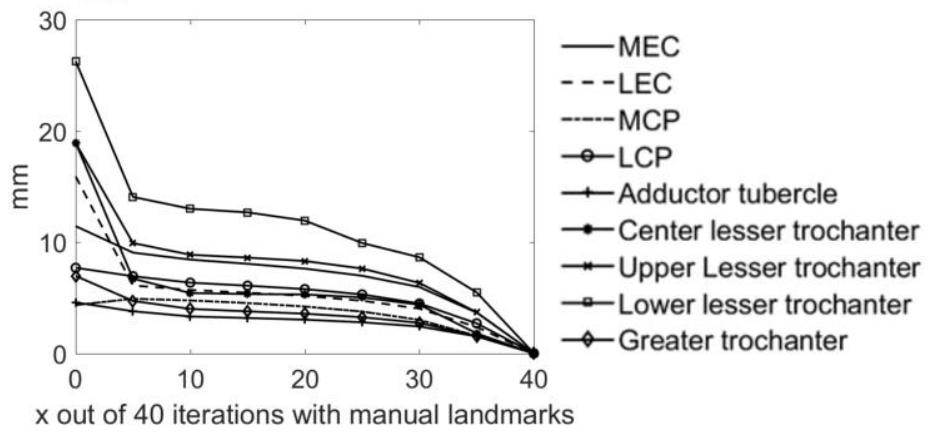

Figure 6. Evolution of A. Geometric error, B. Correspondence quality, C. Mesh quality, D. Behaviour of each landmark in case of combining manually and automatically detected corresponding points in femur. 
A.

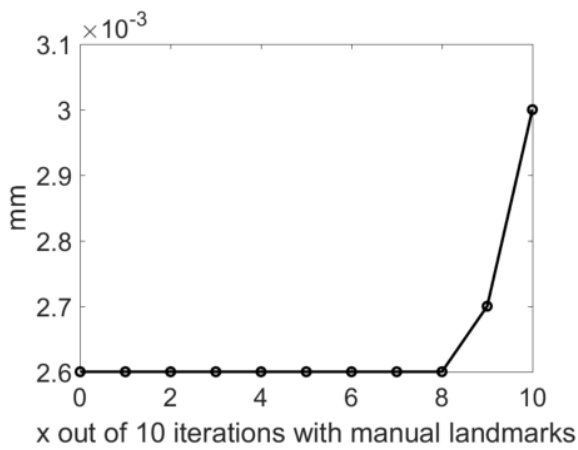

C.

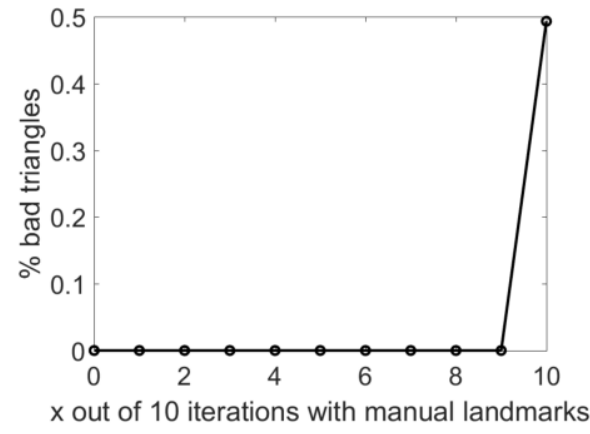

B.

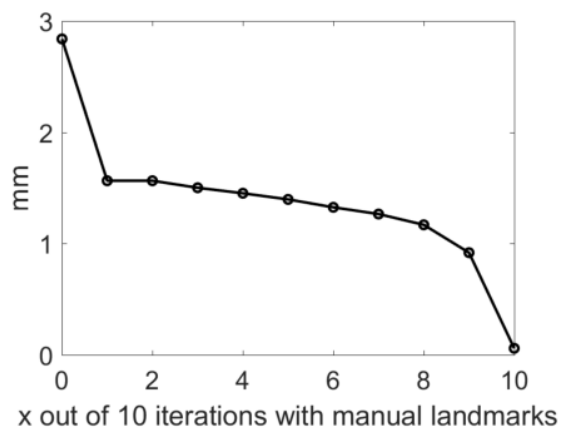

D.

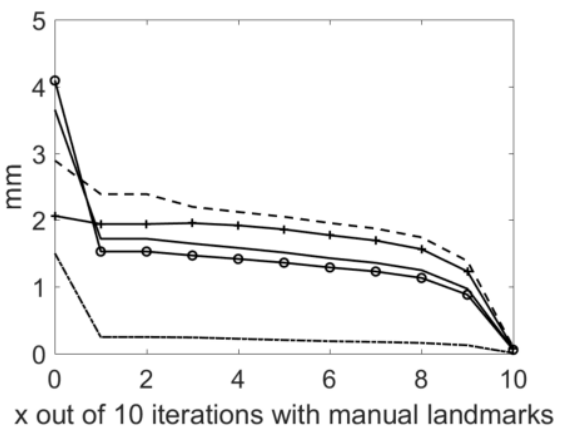

- ACJ dorsal

- - -SCJ ventral

-----Conoid tubercle

$\rightarrow$ ACJ ventral

$\rightarrow$ SCJ inferior

Figure 7. Evolution of A. Geometric error, B. Correspondence quality ,C. Mesh quality, D. Behaviour of each landmark in case of combining manually and automatically detected corresponding points in clavicle.

Table 1. Comparison of correspondence quality of 9 femur landmarks (medial epicondyle (MEP), lateral epicondyle (LEP), most posterior point of medial and lateral condyle (MCP and LCP), the adductor tubercle (AT), the lesser trochanter (LTC), the lesser trochanter upper and lower transition (ULT and LLT), and the upper side of the greater trochanter (GT)) using only corresponding points and the combination of manually and automatically indicated corresponding points.

\begin{tabular}{lccccccccc}
\hline Algorithm & MEC & LEC & MCP & LCP & AT & LTC & ULT & LLT & GT \\
\hline $\begin{array}{l}\text { Automatic } \\
\text { corresponding point } \\
\text { detection [mm] }\end{array}$ & 11.4 & 15.9 & 4.4 & 7.7 & 4.6 & 18.9 & 18.9 & 26.3 & 7.0 \\
$\begin{array}{l}\text { 20 out of 40 iterations } \\
\text { with manually }\end{array}$ & 7.6 & 5.2 & 4.2 & 5.8 & 3.0 & 5.3 & 8.3 & 11.9 & 3.6 \\
$\begin{array}{l}\text { identified landmarks } \\
{[\mathrm{mm} \text { [ }}\end{array}$ & & & & & & & & & \\
\hline
\end{tabular}

